\title{
The quasi-symmetric side of gravity modelling
}

\author{
François Bavaud \\ Section d'Informatique et de Méthodes Mathématiques, Faculté des Lettres, University of \\ Lausanne, CH-1015, Switzerland; e-mail: Francois.Bavaud@imm.unil.ch \\ Received 5 April 2001; in revised form 29 August 2001
}

\begin{abstract}
Many properties of gravity models are sole consequences of the quasi-symmetry condition or its avatars. We investigate here quasi-symmetry per se, in contrast to geographical tradition, which has been more focused on the exogenous socioeconomic and spatial conditions. In particular, the 'size - utility-accessibility' parameterization of migration counts turn out to rely exclusively upon the quasi-symmetry of flows. Various facets of quasi-symmetry are presented and put in correspondence with Markov chains theory, Bradley-Terry-Luce decision theory, the Weidlich-Haag model, and alternative classical statistical models (marginal homogeneity, symmetry, independence). Existing as well as presumably new estimation and model selection procedures (maximum likelihood, minimum discrimination information, maximum entropy, generalized power divergence, least squares and logarithmic least squares) are discussed in a way which unifies different traditions in gravity modelling.
\end{abstract}

\section{Introduction}

Numerous authors (for example, see Berger and Snell, 1957; Theil, 1972, page 281; Bishop et al, 1975, page 287; Andersen, 1990, page 323) have observed various spatial flows, mobility tables, or opinion-shift data $n_{j k}$ to be well described by the quasi-symmetric model, $n_{j k}=\alpha_{j} \beta_{k} \gamma_{j k}$, with $\gamma_{j k}=\gamma_{k j}$.

As a matter of fact (theorem 1 below), quasi-symmetry precisely enables us to parameterize flows as $\{n\} \equiv\{\rho, g, u\}$, where $\{\rho\}$ are the trivial size effects, $\{g\}$ are the size-independent accessibilities quantifying the distance-deterrence effect, the symmetric component of migration, and $\{u\}$ are the size-independent utilities quantifying the places' attractivities or push - pull drive, the antisymmetric component of migration.

The above ingredients are those of gravity modelling, or more precisely constitute its endogenous aspect (the quasi-symmetry condition). Its exogenous aspect relates (typically by a multiple regression) the accessibilities $\{g\}$ to travel costs between places, on one hand, and the utilities $\{u\}$ to local socioeconomic conditions, on the other hand.

In this paper, I seek to derive or to reveal utility and accessibility parameters from the observation of flows, in the same sense that the sole observation of purchases for a given budget level reveals consumers' utility. As such, this approach is thus also of potential relevance for other nonspatial domains such as the study of mobility tables or opinion shifts. My study is endogenous in that I limit myself to study the theoretical properties of the parameters $\{g, u\}$ so defined. I do not address the predictive, exogenous part of the program, namely the confrontation of the parameters with socioeconomic and effective distance data.

More general, non-quasi-symmetric gravity models can indeed be considered, as, for example, in the work of Sen and Smith (1995), where the symmetry assumption on the accessibilities $\{g\}$ is dropped. Although perfectly acceptable in a traditional predictive approach, and in particular testable (Smith, 1984), such an enlargement of the class of models under consideration is not feasible in the revealed approach: without quasi-symmetry, the 'revealed' parameterization $\{\rho, g, u\}$ of flows does not hold any more, and the question of how to construct a mathematically rigorous, behaviorally interpretable parameterization in the general, non-quasi-symmetric case remains open. 
The parameter-identifiability question is not the only issue on which spatial interaction analysis could benefit substantially from accurately identifying the quasi-symmetric component in gravity modelling:

1. Typical gravity features such as the transitivity of flows (Smith and Clayton, 1978) or the absence of aggregation invariance (Schwab and Smith, 1985) are consequences of the quasi-symmetry condition only, irrespective of the exogenous conditions; making the former explicit would thus help characterize, classify, and maybe generate new spatial interaction models (see section 2.3).

2. Splitting gravity calibration into a first phase devoted to the quasi-symmetric estimation of flows followed by the adjustment of utility or mobility parameters relative to the exogenous variables would permit us to determine if an observed violation of the gravity model (4) below is a result of the nonobservation of the endogenous condition, or of the exogenous condition, or both.

3. Identifying the quasi-symmetric component (when present) in various interaction theories constitutes a good starting point for comparing those theories and assessing their differences and resemblances. For instance, Wilson's (1974) maximum entropy approach to gravity modelling automatically insures quasi-symmetry of the predicted flows (theorem 4 below); the Weidlich - Haag interregional migration model (Haag and Weidlich, 1984; Weidlich and Haag, 1988), presented by its contributors as an alternative to traditional gravity modelling, turns out to contain an implicit quasi-symmetric assumption (see section 4).

4. Quasi-symmetry amounts to the reversibility of weights $w_{j k}$ (theorem 1 below; see also Bavaud, 1998), that is, to the phenomenological identity between the Markov process and its time-inverted associated process. Analogous results also hold for more general 'interactive' Markov processes (Smith and Hsieh, 1997a), where the stability of the stationary flows is guaranteed again by quasi-symmetry (Smith and Hsieh, 1997b). Also, quasi-symmetric weights possess real eigenvalues, which considerably simplifies the study of their time evolution, not addressed here.

In section 2 I focus on interregional migration data in relation to the many statistical or behavioral aspects of quasi-symmetry (theorem 1), in particular the $(\rho, g, u)$-parameterization of flows, as well as with their relationship with other models (flows equilibrium, symmetry, and independence: see theorems 2 and 3 below). In section 3 I address the question of approximating non-quasi-symmetric empirical flows by quasi-symmetric parameters, and present different estimation methods (Wilson, 1974; Cressie and Read, 1984; Weidlich and Haag, 1988; Fotheringham and O'Kelly, 1989) under a unifying formalism. In section 4 some aspects of the Weidlich-Haag model are compared with the present work. I have attempted to alleviate the difficulty of a first reading by transferring most of the mathematical details in the appendices.

The paper contains existent material, often fairly scattered in the literature, which I have tried to present in a systematic, self-contained way, as well as new analytic tools and concepts. For the sake of space, short-time properties of quasi-symmetric models, in particular their 'jump' parameterization, eigenvalue decomposition, and their connection to the definition of global mobility indices, are deferred to another paper.

\section{Quasi-symmetric flow and its parameterization}

\subsection{Empirical and stationary profiles}

Flow data consist of the $(m \times m)$ contingency table, $n_{j k}(T)$, counting the number of units (people, goods, or news) initially $(t=0)$ in region $j$ and finally in region $k$ after time $t=T$; intermediate states for $0<t<T$, if any, are not recorded. The same set of labels is used for departure and arrival regions: $j, k=1, \ldots, m$. Immigration and emigration outside the total region are not considered, nor are aging phenomena 
or birth and death events. Total population is not disaggregated into age, gender, or socioeconomic status subgroups. Finally, exogenous socioeconomic conditions, although not explicitly used here, are assumed to stay constant. Thus the class of models presented here describes conserved flows of featureless, identically built individuals, and pretensions of realism, if any, must necessarily be confined to short observation times $T$.

The export (or import) empirical profiles are given by $\rho_{j}^{*}:=n_{j \bullet} / n_{\bullet \bullet}$ (or $\left.\rho_{k}:=n_{j \bullet} / n_{\bullet \bullet}\right)$. The weight (export) matrix, $w_{j k} \equiv w_{j k}(T):=n_{j k}(T) / n_{j \bullet}(T)$, consists of the normalized pattern of migration profiles; because $w_{j k} \geqslant 0$, and $w_{j \bullet}=1$, the weight matrix is, formally, the transition matrix of a Markov chain. ${ }^{(1)}$ Unless the observation period, $T$, is extremely short, the chain so defined is generally irreducible and aperiodic, and thus ergodic; that is, there exists a unique stationary distribution, $\pi_{j}$, satisfying $\pi_{j} \geqslant 0, \pi_{\bullet}=1$, and $\sum_{j} \pi_{j} w_{j k}=\pi_{k}$. Similarly, the normalized pattern of immigration profiles are the import weights, $w_{k j}^{*}:=n_{j k} / n_{\bullet k}=\rho_{j}^{*} w_{j k} / \rho_{k}$, with stationary distribution $\pi_{k}^{*}$.

By construction, empirical and stationary distributions satisfy

$$
\left.\begin{array}{rlrl}
\sum_{j} \pi_{j} w_{j k} & =\pi_{k}, & \sum_{j} \rho_{j}^{*} w_{j k} & =\rho_{k} \\
\sum_{k} \pi_{k}^{*} w_{k j}^{*} & =\pi_{j}^{*}, & \sum_{k} \rho_{k} w_{k j}^{*}=\rho_{j}^{*} .
\end{array}\right\},
$$

The second equation above shows the import profile, $\rho_{k}$, to be the weighted average of the emigration profiles, $w_{j k}$, over all origins, $j=1, \ldots, m$. Similarly, the last equation above shows the export profile, $\rho_{j}^{*}$, to be the mean immigration profile, $w_{k j}^{*}$, across all destinations, $k=1, \ldots, m$. $^{(2)}$

If the fiction of a constant transition matrix rate,

$$
r_{j k}:=\lim _{t \rightarrow \infty}\left(w_{j k}-\delta_{j k}\right) / t \cong\left[w_{j k}(T)-\delta_{j k}\right] / T,{ }^{(3)}
$$

is taken seriously (at least for analytic, if not realistic, reasons) then the spatial weight matrix $\mathbf{W}(T)$ can be extended to arbitrary time $t \neq T$ by $\mathbf{W}(t):=\exp (\mathbf{R} t)$, where $\mathbf{R}$ is the associated transition matrix rate and the occupation profile $\mathrm{f}_{k}(t)$ at time $t$ can be computed as

$$
\mathrm{f}_{k}(t)=\sum_{j=1}^{m} \rho_{j}^{*} w_{j k}(t),
$$

with the results

$$
\mathrm{f}_{k}(0)=\rho_{k}^{*}, \quad \mathrm{f}_{k}(T)=\rho_{k}, \quad \mathrm{f}_{k}(\infty)=\pi_{k}, \quad \mathrm{f}_{k}(-\infty)=\pi_{k}^{*} .
$$

\subsection{Reversible cinematic: quasi-symmetry and the gravity model}

Newton's law of gravitation expresses the attraction force between two bodies of masses $M_{j}$ and $M_{k}$ at mutual distance $D_{j k}$ as $F_{j k}=G M_{j} M_{k} D_{j k}^{-2}$, where $G$ is the universal gravitation constant. Newton's law results from the conjunction of two distinct statements:

(1) The material used here about Markov chains is standard, and can be found, for example, in Feller (1966), Kemeny and Snell (1967), Collins (1974) or Kijima (1997).

(2) This fact (as well as considerations on rectangular flow matrices, not developed here) has motivated the notations $\rho^{*}$ and $\rho$ as well as the choice of indices.

(3) Here and what follows, the Kronecker delta, $\delta_{j k}$, denotes the $(j, k)$-th component of the unit matrix. 
1. the gravitational force $F_{j k}$ is expressed as $\alpha_{j} \beta_{k} \gamma_{j k}$, where $\alpha_{j}$ depends solely on body $j$, $\beta_{k}$ depends solely on body $k$, and $\gamma_{j k}=\gamma_{k j}$ is symmetric;

2. $\alpha_{j}$ is proportional to the body mass $M_{j}, \beta_{k}$ is proportional to the body mass $M_{k}$, and $\gamma_{j k}$ is inversely proportional to the square of the distance $D_{j k}^{2}$.

Contemporary spatial interaction modelling, as expressed, for example, in the work of Fotheringham and O'Kelly (1989) or Sen and Smith (1995), still refers to the model

$$
\begin{aligned}
n_{j k}(T)=\alpha_{j} & \text { (origin socioeconomic conditions }) \\
\times & \beta_{k}(\text { destination socioeconomic conditions }) \\
& \times \gamma_{j k}(\text { effective origin }- \text { destination distance })
\end{aligned}
$$

as the gravity model; the latter results from the conjunction of two distinct statements: 1. the structural or endogenous condition, that is, the flows $n_{j k}$ are of the form $\alpha_{j} \beta_{k} \gamma_{j k}$, with $\gamma_{j k}=\gamma_{k j}$;

2. the exogenous conditions:

(a) the local exogenous condition: the 'propulsiveness' $\alpha_{j}$ (or 'attractivities' $\beta_{k}$ ) depend solely on characteristics of region $j$ (or $k$ ), such as its density, area, climate, socioeconomic conditions, and so on;

(b) the pair exogenous condition: the 'distance-deterrence function', $\gamma_{j k}$, depends solely on some effective symmetric distance between regions $j$ and $k$, such as the travel length, travel time, travel cost, and so on.

Endogenous condition 1 is known as the quasi-symmetry condition in the statistical literature (for example, see Bishop et al, 1975; Andersen, 1990):

Definition 1: The flow $n_{j k}$ is quasi-symmetric if it can be written as $n_{j k}=\alpha_{j} \beta_{k} \gamma_{j k}$, with $\gamma_{j k}=\gamma_{k j}$.

Pair exogenous condition 2(b) is the only one to be specifically spatial, and thus proper to geography. The three components appearing in the reformulation

$$
\text { gravity model }=\text { quasi-symmetry }+ \text { local exogenous condition }
$$

$$
+ \text { pair exogenous condition }
$$

have received unequal attention: with some exceptions [for example, Willekens (1983) or Nijkamp et al (1985) and references therein], the quasi-symmetry property is hardly alluded to in geography, whereas exogenous conditions 2(a) and 2(b) have been extensively studied in the preference and choice-modelling literature. This state of things may partially be explained by theorem 4 presented below: namely, maximum-entropy gravity modelling involving symmetric effective distances, $d_{j k}$, automatically yields quasi-symmetric flows, $n_{j k}$, thus making the explicit requirement of the quasi-symmetry condition unnecessary.

Quasi-symmetric parameterization $\{\alpha, \beta, \gamma\}$ of flows $\{n\}$ is unique up to a multiplicative vector and a multiplicative constant: $\alpha_{j} \beta_{k} \gamma_{j k}=a_{j} b_{k} c_{j k}$, iff $a_{j}=\alpha_{j} h_{j}$, $b_{k}=d \beta_{k} h_{k}$, and $c_{j k}=\gamma_{j k} /\left(d h_{j} h_{k}\right)$, for some nonzero vector $\boldsymbol{h}$ and nonzero constant $d$.

Theorem 1: The following statements are equivalent (see the proof in appendix):

(a) $n_{j k}$ is quasi-symmetric, that is, representable as $n_{j k} \alpha_{j} \beta_{k} \gamma_{j k}$ for some components $\alpha_{j}, \beta_{k}$, and $\gamma_{j k}$, with $\gamma_{j k}=\gamma_{k j}$;

(b) $n_{j k}$ is representable as $n_{j k}=a_{j} c_{j k}$, with $c_{j k}=c_{k j}$ (or equivalently $n_{j k}$ is representable as $n_{j k}=c_{j k} b_{k}$, with $c_{j k}=c_{k j}$ );

(c) $\ln n_{j k}=\mu+\epsilon_{j}+\eta_{k}+\omega_{j k}$, with $\epsilon_{\bullet}=\eta_{\bullet}=\omega_{j_{\bullet}}=\omega_{\bullet k}=0$, and $\omega_{j k}=\omega_{k j}$;

(d) $n_{j k} n_{k l} n_{l j}=n_{j l} n_{l k} n_{k j}$, for any three (distinct or not) regions $j, k$, and $l$;

(e) $w_{j k} w_{k l} w_{l j}=w_{j l} w_{l k} w_{k j}$, for any three (distinct or not) regions $j, k$, and $l$; 
(f) $n_{j k}$ is representable as $n_{j k}=n_{j k}^{\mathrm{g}} \exp \left(u_{k}-u_{j}\right)$, with $n_{j k}^{\mathrm{g}}:=\left(n_{j k} n_{k j}\right)^{1 / 2}=n_{k j}^{\mathrm{g}}$;

(g) $w_{j k}$ is representable as $w_{j k}=w_{j k}^{\mathrm{g}} \exp \left(v_{k}-v_{j}\right)$, with $w_{j k}^{\mathrm{g}}:=\left(w_{j k} w_{k j}\right)^{1 / 2}=w_{k j}^{\mathrm{g}}$;

(h) the Markov chain is time reversible, that is, $\pi_{j} w_{j k}=\pi_{k} w_{k j}$;

$\left(\mathrm{e}^{\prime}\right) w_{k j}^{*} w_{j l}^{*} w_{l k}^{*}=w_{k l}^{*} w_{l j}^{*} w_{j k}^{*}$, for any three (distinct or not) regions $k, j$, and $l$;

$\left(\mathrm{f}^{\prime}\right) n_{k j}$ is representable as $n_{k j}=n_{j k}^{\mathrm{g}} \exp \left(-u_{k}+u_{j}\right)$, with $n_{j k}^{\mathrm{g}}:=\left(n_{j k} n_{k j}\right)^{1 / 2}=n_{k j}^{\mathrm{g}}$;

$\left(\mathrm{g}^{\prime}\right) w_{k j}^{*}$ is representable as $w_{k j}^{*}=w_{k j}^{* \mathrm{~g}} \exp \left(v_{j}^{*}-v_{k}^{*}\right)$, with $w_{k j}^{* g}:=\left(w_{k j}^{*} w_{j k}^{*}\right)^{1 / 2}=w_{j k}^{* g}$;

$\left(\mathrm{h}^{\prime}\right)$ the dual Markov chain is time reversible, that is, $\pi_{k}^{*} w_{k j}^{*}=\pi_{j}^{*} w_{j k}^{*}$.

Property (f), upon which we shall mainly focus in the sequel, is behavioristically attractive: it permits us to decompose a quasi-symmetric flow, $n_{j k}$, into a symmetric part, $n_{j k}^{\mathrm{g}}=\left(n_{j k} n_{k j}\right)^{1 / 2}$ (the geometric mean of the direct and backward transition counts), and into an antisymmetric part, $\exp \left(u_{k}-u_{j}\right)=1 / \exp \left(u_{j}-u_{k}\right)$, expressing differences in relative preferences, $u_{j}$ and $u_{k}$, for places $j$ and $k$; we shall refer to $u_{j}$ as the utility of region $j$. Utilities, which are defined up to an additive constant, materialize possible departures from symmetry: $u_{j} \equiv c$, iff flows are symmetric (see theorem 3 below). If one imposes the normalization

$$
\sum_{j=1}^{m} u_{j}=0
$$

one finds

$$
u_{j}=\frac{1}{2 m} \sum_{k=1}^{m} \ln \frac{n_{k j}}{n_{j k}}=\frac{1}{2} \ln \frac{\pi_{j}}{\rho_{j}^{*}}-\frac{1}{2 m} \sum_{k=1}^{m} \ln \frac{\pi_{k}}{\rho_{k}^{*}}=\frac{1}{2} \ln \frac{\rho_{j}}{\pi_{j}^{*}}-\frac{1}{2 m} \sum_{k=1}^{m} \ln \frac{\rho_{k}}{\pi_{k}^{*}} .
$$

These equations follow from $n_{k j} / n_{j k}=\rho_{k}^{*} \pi_{j} / \rho_{j}^{*} \pi_{k}=\rho_{j} \pi_{k}^{*} / \rho_{k} \pi_{j}^{*}$, themselves a consequence of the reversibility of $w_{j k}$ and $w_{k j}^{*}$ : utilities provide a measure of the empirical profiles - stationary profiles imbalance. Conversely, and this constitutes a fact notable in itself, quasi-symmetry enables stationary profiles to be expressed in closed form, that is,

$$
\begin{aligned}
\pi_{j} & =n_{j \bullet} \prod_{k=1}^{m}\left(\frac{n_{k j}}{n_{j k}}\right)^{\frac{1}{m}} / \sum_{l=1}^{m} n_{l \bullet} \prod_{k=1}^{m}\left(\frac{n_{k l}}{n_{l k}}\right)^{\frac{1}{m}} \\
& =\frac{\rho_{j}^{*} \exp \left(2 u_{j}\right)}{\sum_{k=1}^{m} \rho_{k}^{*} \exp \left(2 u_{k}\right)}, \\
\pi_{j}^{*} & =n_{\bullet j} \prod_{k=1}^{m}\left(\frac{n_{j k}}{n_{k j}}\right)^{\frac{1}{m}} / \sum_{l=1}^{m} n_{\bullet l} \prod_{k=1}^{m}\left(\frac{n_{l k}}{n_{k l}}\right)^{\frac{1}{m}} \\
& =\frac{\rho_{j} \exp \left(-2 u_{j}\right)}{\sum_{k=1}^{m} \rho_{k} \exp \left(-2 u_{k}\right)} .
\end{aligned}
$$

The symmetric part, $n_{j k}^{\mathrm{g}}$, defined in condition (f) contains both size and distancedeterrence (dependency) effects. To disentangle them, we define the independence quotient, $q_{j k}:=n_{j k} / n_{j k}^{\text {th }}=n_{j k} n / n_{j \bullet} n_{\bullet k}=n_{j k} / n \rho_{j}^{*} \rho_{k}$. Then

$$
n_{j k}^{\mathrm{g}}=\left(\frac{n_{j k}}{n_{j k}^{\text {th }}} \frac{n_{k j}}{n_{k j}^{\text {th }}}\right)^{1 / 2}\left(n_{j k}^{\text {th }} n_{k j}^{\text {th }}\right)^{1 / 2}=n\left(q_{j k} q_{k j}\right)^{1 / 2}\left(\rho_{j}^{*} \rho_{j}\right)^{1 / 2}\left(\rho_{k}^{*} \rho_{k}\right)^{1 / 2},
$$

and therefore, as announced,

$$
n_{j k}=\underbrace{n}_{\text {absolute size }} \underbrace{\rho_{j}^{\mathrm{g}} \rho_{k}^{\mathrm{g}}}_{\text {relative size }} \underbrace{g_{j k}}_{\text {distance deterrence }} \underbrace{\exp \left(u_{k}-u_{j}\right)}_{\text {push-pull }},
$$


where

$$
\left.\begin{array}{l}
\rho_{j}^{\mathrm{g}}:=\left(\rho_{j}^{*} \rho_{j}\right)^{1 / 2}(\text { geometric relative size }), \\
g_{j k}:=\left(q_{j k} q_{k j}\right)^{1 / 2}=g_{k j}=q_{j k}^{\mathrm{g}} \text { (pairwise accessibility). }
\end{array}\right\}
$$

The accessibilities, $g_{j k}$, provide the size-independent parameterization revealed by the symmetrized flow: $g_{j k}>1$, iff regions $j$ and $k$ exchange more units than expected by the independence model, that is, iff the relative accessibility between regions $j$ and $k$ is higher than average.

Conditions (a) or (b) of theorem 1 introduce the concept of quasi-symmetry in most expositions; condition (c) constitutes its log-linear formulation (for example, see Christensen, 1990). Condition (d) mirrors the so-called Kolmogorov condition (e), which makes the proportion of units carried in a closed loop independent of the orientation of the loop. Equivalently, the time-reversibility condition for weights (h) in the theory of Markov chains (for example, see Feller, 1966) states that direct $w_{j k}$ and time-reversed, $\hat{w}_{j k}:=\pi_{k} w_{k j} / \pi_{j}$, transition matrices are identical, thus making the direction of time indiscernible. The equivalence

quasi-symmetry of flows $\Leftrightarrow$ reversibility of weights

has been noted by an anonymous referee in McCullagh (1982). Conditions $\left(\mathrm{e}^{\prime}\right)$ to $\left(\mathrm{h}^{\prime}\right)$ reproduce the same properties for reversed flows, $n_{k j}$, or import weights, $w_{k j}^{*}$.

Under time reversal, $t \rightarrow-t$, parameters transform as $u \rightarrow-u$ [compare, for example, conditions (f) and ( $\left.\left.\mathrm{f}^{\prime}\right)\right], g \rightarrow g, \rho^{*} \rightarrow \rho, \rho \rightarrow \rho^{*}$, and $\rho^{\mathrm{g}} \rightarrow \rho^{\mathrm{g}}$. Parameters $v$ and $v^{*}$ appearing in conditions $(\mathrm{g})$ and $\left(\mathrm{g}^{\prime}\right)$ do not possess comparable simple transformation laws (see also section 4).

\subsection{Other models}

Further properties and parameterization $\{\rho, g, u\}$ of quasi-symmetric (QS) flows are best understood in comparison with other models, quasi-symmetric or not. First, flow equilibrium describes the situation where no net transfer of units occurs during the observation time $T$ :

Definition $2(M H)$ : The flow, $n_{j k}$, is in equilibrium if $\rho_{j}^{*}=\rho_{j}$, for all $j=1, \ldots, m$; this situation is referred to as the marginal homogeneity condition in the statistical literature (for example, see Bishop et al, 1975; Andersen, 1990).

Marginal homogeneity can hold or not irrespective of quasi-symmetry: that is, the gravity model can indifferently describe equilibrium as well as nonequilibrium situations. By contrast, the symmetric, independent, and (for completeness' sake) trivial models, defined below, constitute particular quasi-symmetric models:

Definition 3 (SYM): The flow, $n_{j k}$, is symmetric if $n_{j k}=n_{k j}$.

Definition 4 (IND): The flow, $n_{j k}$, is independent if $n_{j k}=\alpha_{j} \beta_{k}$.

Definition 5 (TRI): The flow, $n_{j k}$, is trivial if $n_{j k}=\alpha_{j} \alpha_{j}$.

Figure 1 summarizes the structural relationship between models, in particular the equivalences QS + MH $=$ SYM (Caussinus, 1966; Bishop et al, 1975) and $\mathrm{SYM}+\mathrm{IND}=$ TRI.

The following theorems characterize the models in terms of their empirical and stationary distributions, on the one hand, and in terms of their utility-accessibility parameters, on the other; parallel characterization in terms of weights properties can be found in Bavaud (1998). 


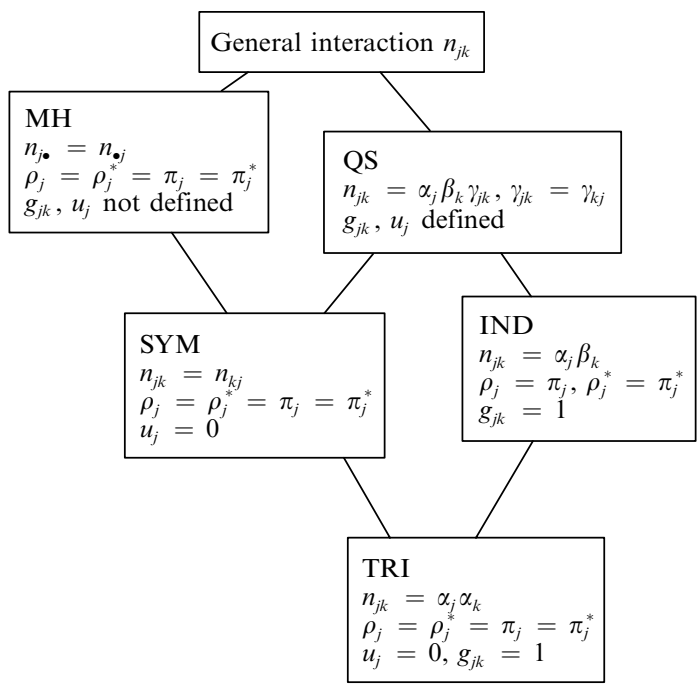

Figure 1. Nesting relationship between models for flows, and their associated parameters and profiles properties. Models are increasingly structured downwards. The bottom models inherit the properties of linked top models.

\section{Theorem 2:}

1. $(Q S) \Rightarrow \rho_{j}^{*} \rho_{j}=c \pi_{j}^{*} \pi_{j}$, for some $c>0, \forall j=1, \ldots, m$;

2. $(M H) \Leftrightarrow \rho_{j}=\rho_{j}^{*}=\pi_{j}=\pi_{j}^{*}, \forall j$;

3. $(S Y M) \Rightarrow \rho_{j}=\rho_{j}^{*}=\pi_{j}=\pi_{j}^{*}, \forall j$;

4. $(I N D) \Rightarrow\left(\rho_{j}=\pi_{j}\right.$ and $\left.\rho_{j}^{*}=\pi_{j}^{*}\right), \forall j$.

Moreover, any of the conditions $\rho_{j}^{*}=\rho_{j}, \rho_{j}^{*}=\pi_{j}$, or $\rho_{j}=\pi_{j}^{*}$ implies that the four distributions $\rho^{*}, \rho, \pi$, and $\pi^{*}$ coincide. By contrast, the condition $\rho_{j}=\pi_{j}$ implies $\rho_{j}^{*}=\pi_{j}^{*}$ and vice versa, but not necessarily the coincidence of the four distributions, as illustrated by the independence model. Also, the constant, $c$, relating empirical to stationary distributions for the QS model is 1 for the models MH, SYM, and IND. Finally, models MH (and thus SYM) and IND are aggregation invariant, as well as QS (Bavaud, 1998).

\section{Theorem 3:}

1. $(S Y M) \Leftrightarrow u_{j} \equiv \operatorname{constant}(=0)$;

2. $(I N D) \Leftrightarrow g_{j k} \equiv \operatorname{constant}(=1)$.

Recall that parameters $\{g, u\}$ are defined for quasi-symmetric flows only: they are not defined for the MH model in general.

\subsection{Behavioral interpretation}

Decomposition (9) can be behaviorally interpreted as follows: a typical individual originally in region $j$ chooses an available opportunity located in destination region $k$ with probability proportional to $g_{j k} \exp \left(u_{k}\right)$. The number of available opportunities in region $k$ is itself proportional to the size, $\rho_{k}^{\mathrm{g}}$, of $k$; thus

$$
\frac{\rho_{k}^{\mathrm{g}} g_{j k} \exp \left(u_{k}\right)}{\sum_{l} \rho_{l}^{\mathrm{g}} g_{j l} \exp \left(u_{l}\right)}=\frac{n_{j k}}{n_{j \bullet}}=w_{j k}
$$

is the probability for a migrant initially in region $j$ to move into region $k$. This simple decision scheme conforms to the Bradley - Terry - Luce (BTL) formalism (for example, 
Luce and Suppes, 1965; Ben-Akiva and Lerman, 1985, pages 43 -55), whose restriction to pairwise choices is expressed as

$$
\begin{aligned}
P_{k k^{\prime}}^{j} & :=\operatorname{prob}\left(\text { a migrant from region } j \text { chooses region } k \text { over region } k^{\prime}\right) \\
& =\frac{w_{j k}}{w_{j k}+w_{j k^{\prime}}}=\frac{1}{1-\exp \left[-\left(U_{k}^{j}-U_{k^{\prime}}^{j}\right)\right]},
\end{aligned}
$$

where

$$
\begin{aligned}
U_{k}^{j} & :=\ln \rho_{k}^{\mathrm{g}}+\ln g_{j k}+u_{k} \\
& =\text { total attractivity of destination } k \text { for a migrant from region } j .
\end{aligned}
$$

The total attractivity, $U_{k}^{j}$ (defined up to an additive constant), additively decomposes into a size attractivity component, $\ln \rho_{k}^{\mathrm{g}}$, measuring the opportunities availability, a proximity attractivity component, $\ln g_{j k}$, betraying the distance deterrence, and an intrinsic utility, $u_{j}$, component taking into account all remaining size-independent and distance-independent effects. By construction, $w_{j k} \geqslant w_{j k^{\prime}}$, iff $U_{k}^{j} \geqslant U_{k^{\prime}}^{j}$. While one certainly expects $g_{j j} \geqslant g_{j k}$, for all $k \neq j$, one could well observe $w_{j j}<w_{j k}$, if region $j$ is simultaneously sufficiently small, close, and unattractive with regard to region $k$, so that, $\ln \rho_{j}^{\mathrm{g}}+\ln g_{i j}+u_{j}<\ln \rho_{k}^{\mathrm{g}}+\ln g_{j k}+u_{k}$.

The last formula in equation (12) is one of the many equivalent forms of the Bradley-Terry-Luce model, namely the binary logit parameterization (for example, Ben-Akiva and Lerman, 1985, pages 70-72). Another equivalent condition is the product rule, $P_{k k^{\prime}}^{j} P_{k^{\prime} k^{\prime \prime}}^{j} P_{k^{\prime \prime} k}^{j}=P_{k k^{\prime \prime}}^{j} P_{k^{\prime \prime} k^{\prime}}^{j} P_{k^{\prime} k}^{j}$ (Luce and Suppes, 1965, page 341). Despite its analogy to conditions (d) or (e) of theorem 1, the product rule is a consequence of equation (12) solely, irrespective of the possible quasi-symmetric nature of $w_{j k}$.

\section{Poisson-like quasi-symmetric models and their calibration}

\subsection{Poisson models}

Real observed flows, $n_{j k}$, never exactly obey the quasi-symmetry condition, $n_{j k}=\alpha_{j} \beta_{k} \gamma_{j k}$; thus quasi-symmetry qualifies at best theoretical parameters, $\lambda$, associated with a probabilistic model defined by a distribution of the form $\mathrm{P}(n \mid \lambda)$, where $n \equiv\left\{n_{j k}\right\}$ stands for the $m^{2}$ complete observed flows. Postulating, in addition, independence between identically distributed individual migratory decisions, one gets the complete Poisson model with parameters $\lambda \equiv\left\{\lambda_{j k}\right\}$ :

$$
\mathrm{P}_{\text {Poisson }}(n \mid \lambda):=\prod_{j, k=1}^{m} \exp \left(-\lambda_{j k}\right) \frac{\lambda_{j k}^{n_{j k}}}{n_{j k} !} .
$$

In view of the well-known Poisson property, $\mathrm{E}\left(n_{j k}\right)=\lambda_{j k}$, it is natural to require the quasi-symmetry property to hold for the $\lambda_{j k}$, that is, to impose the $\frac{1}{2}(m-1)(m-2)$ constraints, $\lambda_{j k}=a_{j} c_{j k}$, with $c_{j k}=c_{k j}$. From now on, the notation $\lambda_{j k}$ will denote the quasi-symmetric theoretical flows, as opposed to the empirical ones, $n_{j k}$.

Export and import theoretical weights, $w_{j k}$ and $w_{k j}^{*}$, as well as the cell distribution, $p_{j k}$, defined below, occur naturally from equation (14) when constraining (in order) the origin outflows, the destination inflows, and the total flow. Indeed, if count configurations, $n=\left\{n_{j k}\right\}$, are restricted to a fixed row total, $n_{j \bullet}$, for each origin $j=1, \ldots, m$, then, using Bayes rule together with

$$
\mathrm{P}_{\text {Poisson }}\left(\left\{n_{j \bullet}\right\} \mid \lambda\right)=\prod_{j=1}^{m} \frac{1}{n_{j \bullet} !} \prod_{k=1}^{m} \exp \left(-\lambda_{j k}\right) \lambda_{j \bullet}^{n_{j k}}=\prod_{j}^{m} \frac{1}{n_{j \bullet} !} \exp \left(-\lambda_{j \bullet}\right) \lambda_{j \bullet}^{n_{\bullet}}
$$


operates the transformation of the Poisson model (14) into the fixed outflows model:

$$
\mathrm{P}_{\text {fixed outflows }}\left(n \mid \lambda,\left\{n_{j \bullet}\right\}\right)=\frac{\mathrm{P}_{\text {Poisson }}(n \mid \lambda)}{\mathrm{P}_{\text {Poisson }}\left(\left\{n_{j \bullet}\right\} \mid \lambda\right)}=\prod_{j=1}^{m} n_{j \bullet} ! \prod_{k=1}^{m} \frac{w_{j k}^{n_{j k}}}{n_{j k} !}, \quad \text { with } w_{j k}:=\frac{\lambda_{j k k}}{\lambda_{j \bullet}} .
$$

Analogously, when count configurations, $n$, are restricted to a fixed column margin, $n_{\bullet k}$, for each destination $k=1, \ldots, m$, the Poisson model (14) then turns into the fixed inflows model:

$$
\mathrm{P}_{\text {fixed inflows }}\left(n \mid \lambda,\left\{n_{\bullet k}\right\}\right)=\prod_{k=1}^{m} n_{\bullet} ! \prod_{j=1}^{m} \frac{w_{j k}^{* n_{j k}}}{n_{j k} !}, \quad \text { with } w_{k j}^{*}:=\frac{\lambda_{j k}}{\lambda_{\boldsymbol{}_{k}}} .
$$

Next, the Poisson model turns into the multinomial model when the total flow, $n_{\bullet \bullet}$, is fixed:

$$
\mathrm{P}_{\text {multinomial }}\left(n \mid \lambda, n_{\bullet \bullet}\right)=n_{\bullet \bullet} ! \prod_{j, k=1}^{m} \frac{p_{j k}^{n_{j k}}}{n_{j k} !}, \quad \text { with } p_{j k}:=\frac{\lambda_{j k}}{\lambda_{\bullet \bullet}} .
$$

Finally, the fixed inflows and outflows model has both margins $\left\{n_{\boldsymbol{}_{\bullet}}\right\}$ and $\left\{n_{\bullet k}\right\}$ fixed. The resulting model is a noncentral hypergeometric distribution [for example, see McCullagh and Nelder (1983) or Agresti (1990) and references therein], whose functional expression is rather awkward. In the simplest case, $m=2$, the knowledge of a single cell (say $n_{11}$ ) suffices in determining the remaining ones (by $n_{12}=n_{1} \bullet-n_{11}$, $n_{21}=n_{\bullet 1}-n_{11}$, and $\left.n_{22}=n_{\bullet 2}-n_{1} \bullet+n_{11}\right)$. The resulting distribution is then

$$
\mathbf{P}_{\text {noncentral hypergeometric }}\left(n_{11} \mid \lambda,\left\{n_{j \bullet}\right\},\left\{n_{\bullet k}\right\}\right)=\frac{\left(\begin{array}{c}
n_{1 \bullet} \\
n_{11}
\end{array}\right)\left(\begin{array}{c}
n_{2} \\
n_{\bullet 1}-n_{11}
\end{array}\right) \theta^{n_{11}}}{\sum_{m=a}^{b}\left(\begin{array}{c}
n_{1 \bullet} \\
m
\end{array}\right)\left(\begin{array}{c}
n_{2} \bullet \\
n_{\bullet 1}-m
\end{array}\right) \theta^{m}},
$$

where $a:=\max \left(0, n_{1} \bullet-n_{\bullet 2}\right)$, and $b:=\min \left(n_{1 \bullet}, n_{\bullet 1}\right)$. The noncentrality parameter, $\theta$, stands for the odds ratio, $\theta=\lambda_{11} \lambda_{22} / \lambda_{12} \lambda_{21}$. Equation (19) turns into the ordinary hypergeometric distribution when $\theta=1$.

\subsection{Parameter estimation}

We now expose the motivations and computational details of the main approaches governing the determination of quasi-symmetric theoretical complete flows, $\lambda_{j k}$ (or their partial descriptions $w_{j k}, w_{k j}^{*}$, or $p_{j k}$ ), from empirical ones, $n_{j k}$. Numerous methods have been used by many authors for different purposes in various contexts; I seek to unify them by presenting six procedures, all defining $\lambda=\left\{\lambda_{j k}\right\}$ as the best quasisymmetric approximations of the observed flows, $n=\left\{n_{j k}\right\}$, that is, as the solution of the minimization problem

$$
\underset{\lambda ; \lambda \text { quasi-symmetric }}{\operatorname{minimize}} F(\lambda, n),
$$

where $F(\lambda, n) \geqslant 0$ is a nonnegative dissimilarity functional satisfying $\mathrm{F}(\lambda, n)=0$, iff $\lambda=n$, that is, iff the flows, $n_{j k}$, are quasi-symmetric. Associated proofs and mathematical details can be found in the appendix. A summary is given in table 1 (see over). (A) Maximum-likelihood estimates, $\lambda^{\mathrm{ML}}$, of the parameters, $\lambda=\left\{\lambda_{j k}\right\}$, are solutions to the problem

$$
\underset{\lambda ; \lambda \text { quasi-symmetric }}{\operatorname{maximize}} \mathrm{P}_{\text {Poisson }}(n \mid \lambda)=\mathrm{P}_{\mathrm{Posson}}\left(n \mid \lambda^{\mathrm{ML}}\right),
$$


Table 1. Some properties of the estimates.

\begin{tabular}{|c|c|c|c|c|c|c|}
\hline \multirow[t]{2}{*}{ Property } & \multicolumn{6}{|l|}{ Estimate $^{\mathrm{a}}$} \\
\hline & $s>0$ & $s<0^{\mathrm{b}}$ & $s=0^{\mathrm{c}} \mathrm{ML}$ & $M E^{c}$ & LS & LLS \\
\hline$\lambda_{j j}=n_{j j}$ & $\begin{array}{l}\text { NO } \\
\text { (negative bias) }\end{array}$ & $\begin{array}{l}\text { NO } \\
\text { (positive bias) }\end{array}$ & yes & no & yes & yes \\
\hline$\lambda_{j \bullet}=n_{j \bullet}, \lambda_{\bullet k}=n_{\bullet k}$ & no & no & yes & yes & no & no \\
\hline$\lambda_{\text {e. }}=n_{\text {.. }}$ & yes & yes & yes & yes & no & no \\
\hline Zero-flows consistency & yes & no & yes & yes & yes & no \\
\hline
\end{tabular}

a ML, maximum likelihood; ME, maximum entropy; LS, least squares; LLS, logarithmic least squares. $s$-estimates are the generalized power divergence estimates, including ML $(s=0)$ and MDI $(s=-1)$.

$\mathrm{b}$ Including MDI, maximum discrimination information.

c Under fixed margins and trace.

and satisfy (see appendix)

$$
\lambda_{j}^{\mathrm{ML}}=n_{j \bullet}, \quad \lambda_{j k}^{\mathrm{ML}}+\lambda_{k j}^{\mathrm{ML}}=n_{j k}+n_{k j} .
$$

Instead of working with counts, cell distributions,

$$
\begin{aligned}
& \left.p_{j k}=\frac{\lambda_{j k}}{\lambda_{\bullet \bullet}} \text { (theoretical cell distribution }\right), \\
& f_{j k}:=\frac{n_{j k}}{n_{\bullet \bullet}} \text { (empirical cell distribution), }
\end{aligned}
$$

can also be used. As a matter of fact (see appendix), the solution, $\lambda_{j k}^{\mathrm{ML}}$, of problem (21) can be expressed as

$$
\lambda_{j k}^{\mathrm{ML}}=n_{\bullet \bullet} p_{j k}^{\mathrm{ML}},
$$

where $p^{\mathrm{ML}}$ is the solution of the minimum-divergence problem

$$
\underset{p ; p \text { quasi-symmetric }}{\operatorname{minimize}} \mathrm{K}(f \| p)=\mathrm{K}\left(f \| p^{\mathrm{ML}}\right)=: K^{\mathrm{ML}} .
$$

Here and in the rest of this paper, $\mathrm{K}(g|| h):=\sum_{j k} g_{j k} \ln g_{j k} / h_{j k}$ is the Kullback - Leibler divergence between the cell distributions, $g$ and $h$ (obeying $g_{j k}, h_{j k} \geqslant 0$, and $g_{\text {.• }}=h_{\bullet \bullet}=1$ ).

(B) The minimum-discrimination-information procedure (Kullback, 1959) consists in estimating parameters $\lambda_{j k}$ as ${ }^{(4)}$

$$
\lambda_{j k}^{\mathrm{MDI}}=n_{\bullet \bullet} p_{j k}^{\mathrm{MDI}},
$$

where $p^{\mathrm{MDI}}$ is the solution to the problem

$$
\underset{p ; p \text { quasi-symmetric }}{\operatorname{minimize}} \mathrm{K}(p \| f)=\mathrm{K}\left(p^{\mathrm{MDI}} \| f\right)=: K^{\mathrm{MDI}} .
$$

They satisfy (see appendix)

$$
\left.\begin{array}{rl}
\sum_{k} \lambda_{j k}^{\mathrm{MDI}} \ln \frac{\lambda_{j k}^{\mathrm{MDI}}}{n_{j k}}=K^{\mathrm{MDI}} \lambda_{j \bullet}^{\mathrm{MDI}}, \\
\lambda_{j k}^{\mathrm{MDI}} \ln \frac{\lambda_{j k}^{\mathrm{MDI}}}{n_{j k}}+\lambda_{k j}^{\mathrm{MDI}} \ln \frac{\lambda_{k j}^{\mathrm{MDI}}}{n_{k j}}=K^{\mathrm{MDI}}\left(\lambda_{j k}^{\mathrm{MDI}}+\lambda_{k j}^{\mathrm{MDI}}\right) .
\end{array}\right\}
$$

(4) Some authors call MDI-estimates what we call here ML-estimates (Fotheringham and O'Kelly, 1989, page 60), and MMDI-estimates our MDI-estimates (Bishop et al, 1975, page 346). 
(C) Maximum-entropy estimation (Jaynes, 1957; Wilson, 1974) determines a theoretical distribution $p^{\mathrm{ME}}$ solution of

$$
\underset{p ; p \text { quasi-symmetric; }\left\langle A^{\alpha}\right\rangle_{p}=\left\langle A^{\alpha}\right\rangle_{f}}{\operatorname{minimize}}\left(p \| p^{0}\right)=\mathrm{K}\left(p^{\mathrm{ME}} \| p^{0}\right) .
$$

Here $p^{0}$ stands for a prior distribution representing a first, fairly rough, guess of the distribution expected by the researcher. Furthermore, and more specifically, the researcher can in addition compel the theoretical average values of a set of $q$ quantities, $A_{j k}^{\alpha}$, to coincide with the empirical average values observed in the data, namely to fix the $q$ constraints

$$
\sum_{j k} A_{j k}^{\alpha} p_{j k}=\sum_{j k} A_{j k}^{\alpha} f_{j k}, \quad \text { for } \alpha=1, \ldots, q .
$$

In the absence of the quasi-symmetric constraint, $p^{\mathrm{ME}}$, is well known to be of the form (for example, see Cover and Thomas, 1991, page 267):

$$
p_{j k}^{\mathrm{ME}}=\exp \left(\mu_{0}+\sum_{\alpha=1}^{q} \mu_{\alpha} A_{j k}^{\alpha}\right) p_{j k}^{0},
$$

where $\mu_{0}, \mu_{1}, \ldots, \mu_{q}$ are real numbers chosen so that $p^{\mathrm{ME}}$ satisfies the constraints, namely

$$
\sum_{j k} p_{j k}^{\mathrm{ME}}=1, \quad \text { and } \sum_{j k} p_{j k}^{\mathrm{ME}} A_{j k}^{\alpha}=\sum_{j k} f_{j k} A_{j k}^{\alpha}, \quad \forall \alpha .
$$

(D) Generalized-power-divergence minimization (Cressie and Read, 1984) leads to an estimate of parameters as

$$
\lambda_{j k}^{s}=n_{. .} p_{j k}^{s},
$$

where $p^{s}$ is the solution to the problem

$$
\underset{p ; p \text { quasi-symmetric }}{\operatorname{minimize}} \mathbf{I}_{s}(f: p)=\mathbf{I}_{s}\left(f: p^{s}\right)=: \mathbf{I}_{s},
$$

and where $\mathrm{I}_{s}(g: h):=[s(s+1)]^{-1} \sum_{j k} g_{j k}\left[\left(g_{j k} / h_{j k}\right)^{s}-1\right]$ is the generalized-power-divergence functional, indexed by the real number $s$. Using $\lim _{x \rightarrow 0}\left(a^{x}-1\right) / x=\ln a$, for $a>0$, shows that $\mathrm{I}_{0}(g: h)=\mathrm{K}(g \| h)$, and $\mathrm{I}_{-1}(g: h)=\mathrm{K}(h \| g)$. Thus the generalized power divergence generalizes both the ML and the MDI procedures. The parameters satisfy (see appendix)

$$
\sum_{k} n_{j k}\left(\frac{n_{j k}}{\lambda_{j k}^{s}}\right)^{s}=B_{s} \lambda_{j \bullet}^{s}, \quad n_{j k}\left(\frac{n_{j k}}{\lambda_{j k}^{s}}\right)^{s}+n_{k j}\left(\frac{n_{k j}}{\lambda_{k j}^{s}}\right)^{s}=B_{s}\left(\lambda_{j k}^{s}+\lambda_{k j}^{s}\right),
$$

where $B_{s}:=s(s+1) \mathbf{I}_{s}+1$.

(E) Least squares (LS) estimation consists in minimizing

$$
\underset{\lambda ; \lambda \text { quasi-symmetric }}{\operatorname{minimize}} \sum_{j k}\left(\lambda_{j k}-n_{j k}\right)^{2}=\sum_{j k}\left(\lambda_{j k}^{\mathrm{LS}}-n_{j k}\right)^{2}
$$

Solutions $\lambda_{j k}^{\mathrm{LS}}$ satisfy (see appendix)

$$
\left(\lambda_{j k}^{\mathrm{LS}}\right)^{2}+\left(\lambda_{k j}^{\mathrm{LS}}\right)^{2}=\lambda_{j k}^{\mathrm{LS}} n_{j k}+\lambda_{k j}^{\mathrm{LS}} n_{k j}, \quad \sum_{l=1}^{m}\left(\lambda_{j l}^{\mathrm{LS}}\right)^{2}=\sum_{l=1}^{m} \lambda_{j l}^{\mathrm{LS}} n_{j l} .
$$

This in particular shows the value of the minimum (35) to be $\sum_{j k}\left[n_{j k}^{2}-\left(\lambda_{j k}^{\mathrm{LS}}\right)^{2}\right] \geqslant 0$. (F) Logarithmic least squares (LLS) estimation yields parameters $\lambda^{\text {LLS }}$ solutions of

$$
\underset{\lambda ; \lambda \text { quasi-symmetric }}{\operatorname{minimize}} \sum_{j k}\left(\ln \lambda_{j k}-\ln n_{j k}\right)^{2}=\sum_{j k}\left(\ln \lambda_{j k}^{\mathrm{LLS}}-\ln n_{j k}\right)^{2} .
$$


They satisfy (see appendix)

$$
\lambda_{j k}^{\mathrm{LLS}} \lambda_{k j}^{\mathrm{LLS}}=n_{j k} n_{k j}, \quad \quad \prod_{k=1}^{m} \lambda_{j k}^{\mathrm{LLS}}=\prod_{k=1}^{m} n_{j k} .
$$

The main virtue of LLS estimation is the existence of a closed-form solution (rather than an iterative one as in ML, MDI, and LS-estimation), apparently new, namely

$$
\lambda_{j k}^{\mathrm{LLS}}=\left(n_{j k} n_{k j}\right)^{1 / 2} \prod_{l=1}^{m}\left(\frac{n_{j l} n_{l k}}{n_{k l} n_{l j}}\right)^{\frac{1}{2 m}} .
$$

It is straightforward to verify equation (38) as well as condition (d) of the theorem 1, the latter insuring that parameters defined by equation (39) are quasi-symmetric. Also, $\lambda_{j k}^{\mathrm{LLS}}=n_{j k}$, iff $n_{j k}$ is quasi-symmetric: iterating equation (39) keeps producing the fixed point, $\lambda_{j k}=\lambda_{j k}^{\mathrm{LLS}}$, because $\lambda_{j k}=\left(\lambda_{j k} \lambda_{k j}\right)^{1 / 2}\left(\lambda_{j k} / \lambda_{k j}\right)^{1 / 2}$, and $\left(\lambda_{j l} \lambda_{l k}\right) /\left(\lambda_{k l} \lambda_{l j}\right)=\lambda_{j k} / \lambda_{k j}$, by condition $(\mathrm{d})$ of theorem 1.

\subsection{Comments}

The divergence, $\mathrm{K}(g \| h)$, can be interpreted as the measure of the information gained (or the surprise generated) when the distribution $g$ replaces the prior distribution $h$. Assuming $g$ to represent the true hypothesis, $\mathrm{K}(g \| h)$ also measures the mean information for discrimination in favor of hypothesis $(g)$ against hypothesis $(h)$ (Kullback, 1959). Its main property is $\mathrm{K}(g \| h)=0$, iff $g=h$, and $\mathrm{K}(g \| h)>0$, for $g \neq h$.

Equation (25) thus shows the maximum-likelihood estimate, $p^{\mathrm{ML}}$, to be the prior quasi-symmetric distribution, making the observed data, $f$, as little surprising as possible or, precisely most likely: the likelihood of the data under an (unquestioned) quasi-symmetry assumption is at stake here. Conversely, questioning the quasisymmetric origin of (unquestioned) data, as expressed by equation (27), yields the minimum-discrimination-information estimation, $p^{\mathrm{MDI}}$ : the prior distribution consists here of the data themselves, which now play a model-like role in reference to the classical ML procedure. To that extent, MDI estimation constitutes a rather radical and bold strategy which typically can, as shown later, yield inconsistencies whenever some categories have not been observed $\left(f_{j k}=0\right)$, as in the cases of a small sample or/and small observation period, $T$.

Maximum-entropy estimation can be considered as a flexible (through the freedom of choice of the prior $p^{0}$ and of the constraints $A_{j k}^{\alpha}$ ) and safe (as long as $p_{j k}^{0}>0$ everywhere) extension of MDI estimation; particularly popular is the uniform prior, $p_{j k}^{0}=$ constant. Wilson (1974) showed the different variants of the gravity model to be essentially recoverable from the maximum-entropy formalism involving constraints bearing upon

(1) the average trip length [with $A_{j k}:=d_{j k}$, or, more generally, $A_{j k}:=\mathrm{f}\left(d_{j k}\right)$ ],

(2) the average profile of origins (with $A_{j k}^{\alpha}:=\delta_{j \alpha}$, for $\alpha=1, \ldots, m$ ),

(3) the average profile of destinations (with $A_{j k}^{\alpha}:=\delta_{\alpha k}$, for $\alpha=1, \ldots, m$ ).

Precisely, constraints (1) yield the so-called unconstrained interaction model, constraints

(1) and (2) the production-constrained, constraints (1) and (3) the attraction-constrained and constraints (1), (2), and (3) the doubly constrained interaction model (Wilson, 1974; Fotheringham and O'Kelly, 1989). Other constraints of possible relevance in gravity modelling are

(4) the average proportion of stayers constraint $\left(A_{j k}:=\delta_{j k}\right)$,

(5) the marginal homogeneity constraint $\left(A_{j k}^{\alpha}:=\delta_{j \alpha}-\delta_{\alpha k}\right.$, for $\left.\alpha=1, \ldots, m\right)$.

In contrast to the above constraints, the quasi-symmetry condition, $p_{j k}=a_{j} c_{j k}$, with $c_{j k}=c_{k j}$, is not expressible in the form (30). However, and this constitutes a crucial (although unnoticed) fact for gravity modelling, the quasi-symmetry of the 
maximum-entropy estimate $p^{\mathrm{ME}}$ is automatically satisfied under any of the constraints (1) - (5) above or a combination of them [provided $\mathrm{f}\left(d_{j k}\right)=\mathrm{f}\left(d_{k j}\right)$ ]. More generally, the following, easily proved, result holds:

Theorem 4 (maximum-entropy lemma for quasi-symmetry): if the prior $p_{j k}^{0}$ is quasisymmetric, so is $p_{j k}^{\mathrm{ME}}$ in equation (31), provided the constraints $A_{j k}^{\alpha}$ satisfy $A_{j k}^{\alpha}-A_{k j}^{\alpha}=T_{j}^{\alpha}-T_{k}^{\alpha}$, for some $T_{j}^{\alpha}$, for all $\alpha=1, \ldots, q$.

In my opinion, this lemma explains why maximum-entropy gravity modelling (C) [proposed in geography by Wilson (1974) and following a well-established practice (Jaynes, 1957) initiated in statistical mechanics] did not witness special interest in the identification and study of the quasi-symmetric, endogenous side of gravity in itself. To be complete, it should be added that maximum-entropy formalism is nowadays increasingly recognized as an offspring of central-limit theorems for dependent variables gathered through the theory of large deviations label (for example, Bucklew, 1990) rather than as a first principle: for instance, a rigorous proof of the procedure (31) can be found in Van Campenhout and Cover (1981).

As mentioned, both ML and MDI estimates (A) and (B) are particular cases of the power-divergence estimates $(D), \lambda_{j k}^{s}$, in equation (32). Actually, Cressie and Read (1984) have observed the divergence, $\mathrm{I}_{s}(g: h)$, to yield other well-known functionals for particular values of $s$, namely (in order) the ordinary $\chi^{2}$, the Freeman-Tuckey statistic (or Hellinger dissimilarity) and the Neyman $\chi^{2}$ :

$$
\left.\begin{array}{c}
\mathrm{I}_{1}(g: h)=\frac{1}{2} \sum_{j k} \frac{\left(h_{j k}-g_{j k}\right)^{2}}{h_{j k}}, \quad \mathrm{I}_{-1 / 2}(g: h)=2 \sum_{j k}\left(h_{j k}^{1 / 2}-g_{j k}^{1 / 2}\right)^{2}, \\
\mathrm{I}_{-2}(g: h)=\frac{1}{2} \sum_{j k} \frac{\left(h_{j k}-g_{j k}\right)^{2}}{g_{j k}}
\end{array}\right\}
$$

The statistical properties of $\mathrm{I}_{\mathrm{s}}(g: h)$ (concerning, for example, its robustness or its asymptotic behavior when the null model is false) generally strongly depend upon the value of $s$ : the reader is referred to Cressie and Read (1984) for guidelines about the choice of $s$. However, all power-divergence functionals, $\mathrm{I}_{\mathrm{s}}(g: h)$, are ' $\mathrm{H}_{0}$-equivalent' in that, irrespective of the value of $s$, and provided data $g$ follow the null model $\mathrm{H}_{0}$ expressed by $h$, the quantity $2 n_{\bullet \bullet} \mathrm{I}_{\mathrm{s}}(g: h)$ asymptotically follows a $\chi^{2}$-distribution, with $\frac{1}{2}(m-1)(m-2)$ degrees of freedom when $\mathrm{H}_{0}$ constitutes the quasi-symmetry hypothesis.

In contrast to the procedures (A), (B), and (D), LS and LLS estimates (E) and (F) [whose close variants can be found in Weidlich and Haag (1988)] constitute plain optimal-fit parameters, and do not allow proper model selection. In particular, and contrary to a common practice, the squared Pearson correlation, $r^{2}$, between empirical and estimated counts, whose value is typically close to one ${ }^{(5)}$ should not be interpreted as a goodness-of-fit measure aimed at confirming of the model. A large $r^{2}$ indicates only a strong correlation between observed and estimated values, which is to be expected in virtually any situation but the most desperate.

An important practical issue (particularly for small regions and/or small observation times) is the question of the zero-flows consistency of the estimate, namely the behavior of $\lambda_{j k}$ when a count, $n_{j k}$, is zero. The most threatening situation arises when $n_{j k}=0$, for some off-diagonal component, while $n_{k j}>0$; in this case, generalized divergence estimates for $s<0$ and LLS estimates become inconsistent, in that their associated functionals (33) and (37) become infinite, and makes the minimization (5) For example, $r^{2}=0.998$ for LLS estimates $\left(\lambda_{j k}^{\mathrm{LLS}}\right)$ or see $r^{2} \cong 0.98$ in Weidlich and Haag (1988, pages $45-48$ ) for a comparable problem. 
problem indefinite. A commonly used remedy to the zero-flows problem, although lacking theoretical justification and control, consists in adding one to every null empirical flow.

The marginal estimates, $\lambda_{j \bullet}$ and $\lambda_{\bullet k}$, are generally biased, except for ML estimates, and for ME estimates when explicitly required. Moreover, the bias pattern associated to off-diagonal estimates is systematically antisymmetric for all methods, that is, satisfies $\lambda_{j k}>n_{j k}$, iff $\lambda_{k j}<n_{k j}$, for all $j \neq k$. Finally, $\lambda_{\bullet \bullet}^{s}=n_{\bullet \bullet}$ by construction, but $\lambda_{\bullet \bullet}^{\mathrm{LS}} \geqslant n_{\bullet \bullet}$, and $\lambda_{\bullet \bullet}^{\mathrm{LLS}} \geqslant n_{\bullet \bullet}$; analogous behaviors, explainable in terms of Jensen's or arithmetic mean-geometric mean inequalities, have been noticed in similar contexts (Fotheringham and O'Kelly, 1989, pages 44, 48). Similarly, convexity properties of the generalized power divergence make $\lambda_{j j}^{s} \geqslant n_{j j}$, for $s<0$ (stayers' overestimation), $\lambda_{j j}^{s} \leqslant n_{j j}$, for $s>0$ (stayers' underestimation), and $\lambda_{j j}^{s}=n_{j j}$, for $s=0$.

\section{The Weidlich - Haag model}

Weidlich, Haag, and other authors (Haag and Weidlich, 1984; Weidlich and Haag, 1988) developed a theory of interregional migration inspired from nonequilibrium statistical mechanics (master equation and synergetics). They present their approach, which describes migratory transition rates, $r_{j k}$, rather than migratory transition probabilities, $w_{j k}$, as an alternative to traditional gravity modelling, more general and flexible, and able to deal in particular with nonequilibrium and nonstationary situations. They also propose a utility-mobility parameterization of the transition rates, and work out their statistical estimation by methods very close to (E) and (F) above (LS and LLS estimates). While acknowledging the interest and merit of the Weidlich-Haag approach and the collective work it has inspired in general, I found two points turned problematic; I hope to clarify them in the short discussion below.

(1) I have some doubts about the proclaimed greater generality of the Weidlich-Haag approach with respect to traditional gravity modelling. On one hand, the utilitymobility parameterization used by the authors is based upon the equation (Weidlich and Haag, 1988, page 19)

$$
r_{j k}(t)=v_{j k}(t) \exp \left[v_{k}(t)-v_{j}(t)\right], \quad \text { with } v_{j k}(t)=v_{k j}(t),
$$

which is nothing but the quasi-symmetry condition for instant transition rates, $r_{j k}(t)$. Yet, the authors involved in the Weidlich-Haag approach do not mention the latter constraint as such, but seem instead to consider equation (41) as a universally applicable parameterization. If transition rates, $r_{j k}(t)$ are constant (stationarity), the quasi-symmetry of the latter exactly amounts in the quasi-symmetry of the transition probabilities, $w_{j k}$, as in the classical gravity model. On the other hand, the consideration of time-varying transition rates, $r_{j k}(t)$, of course makes the model more flexible, but exactly the same kind of flexibility can be attained by the gravity model when considering time-varying pull-push and distance-deterrence effects, $u_{j}(t), g_{j k}(t)$. Finally, we have seen (figure 1 and theorem 2) QS and $\mathrm{MH}$ to be independent as models, which implies that gravity models can indifferently tackle equilibrium as well as nonequilibrium situations. In conclusion, as far as parameters estimation and model fitting is concerned, the Weidlich-Haag approach seems very close, if not equivalent, to a purely endogenous gravity modelling (that is, taking into account the quasi-symmetry constraint only). Complete gravity modelling (that is, incorporating in addition exogenous constraints) is therefore bound, as observed, to possess a systematically lower goodness of fit than the Weidlich-Haag model. ${ }^{(6)}$ 
(2) In the stationary case, $r_{j k}(t)=r_{j k}(0)$, the Weidlich-Haag contributors correctly estimate $v_{j}$ in equation (41) $)^{(7)}$ as (Weidlich and Haag, 1988, page 38)

$$
v_{j}=\frac{1}{2 m} \sum_{k=1}^{m} \ln \frac{n_{k j} n_{j \bullet}}{n_{j k} n_{k \bullet}} .
$$

The problem is that the 'utilities', $v_{j}$, so defined are strongly size dependent. In fact, comparison of equations (5) and (42) shows that

$$
v_{j}=u_{j}+\frac{1}{2} \ln n_{j \bullet}-\frac{1}{2 m} \sum_{k=1}^{m} \ln n_{k \bullet},
$$

where $u_{j}$ represent the size-independent utilities. In the Weidlich-Haag approach, the behavioral status of this size effect remained uncertain, ranging from an interpretation in terms of an 'agglomeration - saturation' genuine behavioral effect [" $\ldots$ the population prefers high density living, but only up to a certain level" (Weidlich and Haag, 1988, page 91)] to the status of a purely geometric artifact, leading the authors to compare 'regional preferences', defined as the residuals, $v_{j}-a n_{j \bullet}-b n_{j}^{2}$ (where $a$ and $b$ are adjusted by a multiple regression), rather than utilities, $v_{j}$, themselves. The second interpretation nowadays seems routinely adopted by workers in the domain, ${ }^{(8)}$ and produces 'regional preferences' numerically close to the size-independent utilities, $u_{j}$; however, one can simply point out that using evaluation (5) directly is conceptually and computationally much simpler.

\section{Conclusion}

Modelling spatial flows is a major and durable activity in geography, which has attracted many workers and inspired many methods. Focusing upon quasi-symmetry, as I have done in this paper, permits us to compare and partially unify different styles and traditions, as, for example, expressed in the books of Weidlich and Haag (1988), Fotheringham and O'Kelly (1989), and Sen and Smith (1995). It also permits a sharp discrimination between two sources of possible misfit for gravity models, namely the endogenous misfit (occurring when flows are not quasi-symmetric) and the exogenous misfit (occurring when utilities are not expressible by local socioeconomic conditions, or when accessibilities are not expressible by generalized distances between regions). Also, it helps characterizing and situating the gravity model in relation to other wellknown probabilistic models (Markov chains), statistical models (marginal homogeneity, symmetry, and independence) and behavioral models (the Bradley-Terry-Luce decision scheme). Finally, the quasi-symmetry property allows us to parameterize flows by uniquely defined utilities and accessibilities, whose size invariance makes them comparable across different aggregation levels. The logical next task, namely the comparison of flows with differing observation periods, will be covered in another paper.

Acknowledgements. I am indebted to J B Racine for introducing me to the work of Weidlich, Haag, and others. Stimulating discussions with J Arbet, S Roman, and F Perrot, as well as the challenging comments and helpful suggestions of three anonymous referees are gratefully acknowledged.

\section{References}

Agresti A, 1990 Categorical Data Analysis (John Wiley, New York)

Andersen E B, 1990 The Statistical Analysis of Categorical Data (Springer, Berlin)

Bavaud F, 1998, "Models for spatial weights: a systematic look" Geographical Analysis 30153 - 171

(7) Or, equivalently, $v_{j}$ as defined in statement (g) of theorem 1.

(8) Private communication, Lena Sanders, Equipe P.A.R.I.S., CNRS, University of Paris I. 
Ben-Akiva M, Lerman S R, 1985 Discrete Choice Analysis: Theory and Application to Travel Demand (MIT Press, Cambridge, MA)

Berger J, Snell J L, 1957, "On the concept of equal exchange" Behavioral Science 2111 - 118

Bishop Y M M, Fienberg S E, Holland P W, 1975 Discrete Multivariate Analysis (MIT Press, Cambridge, MA)

Bucklew J A, 1990 Large Deviation Techniques in Decision, Simulation and Estimation (John Wiley, New York)

Caussinus H, 1966, "Contribution statistique à l'analyse des tableaux de corrélation" [Statistical contribution to the analysis of correlation tables] Annales de la Faculté des Sciences de l'Université de Toulouse $2977-182$

Christensen R, 1990 Log-linear models (Springer, Berlin)

Collins L, 1974 An Introduction to Markov Chain Analysis (Geographical Abstracts Ltd, University of East Anglia, Norwich)

Cover T M, Thomas J A, 1991 Elements of Information Theory (John Wiley, New York)

Cressie N, Read T R C, 1984, "Multinomial goodness-of-fit tests" Journal of the Royal Statistical Society B $46440-464$

Feller W, 1966 An Introduction to Probability Theory and Its Applications (John Wiley, New York)

Fotheringham A S, O'Kelly M E, 1989 Spatial Interaction Models: Formulations and Applications (Kluwer, Dordrecht)

Haag G, Weidlich W, 1984, "A stochastic theory of interregional migration" Geographical Analysis $16331-357$

Jaynes E T, 1957, "Information theory and statistical mechanics" Physical Review 106 620 - 630; $108171-190$

Kemeny J G, Snell J L, 1967 Finite Markov Chains (D Van Nostrand, Princeton, NJ)

Kijima M, 1997 Markov Processes for Stochastic Modelling (Chapman and Hall, London)

Kullback S, 1959 Information Theory and Statistics (John Wiley, New York)

Luce R D, Suppes P, 1965, "Preference, utility, and subjective probability", in Handbook of Mathematical Psychology, Volume 3 Eds R D Luce, R R Bush, E Galanter (John Wiley, New York) pp $249-410$

McCullagh P, 1982, "Some applications of quasi-symmetry" Biometrika 69303 - 308

McCullagh P, Nelder J A, 1983 Generalized Linear Models (Chapman and Hall, New York)

Nijkamp P, Leitner H, Wrigley N (Eds), 1985 Measuring the Unmeasurable (Martinus Nijhoff, Dordrecht)

Pumain D, 1988, "France”, in International Migration Eds W Weidlich, G Haag (Springer, Berlin) pp $148-153$

Schwab M G, Smith T R, 1985, "Functional invariance under spatial aggregation from continuous spatial interaction models" Geographical Analysis 17 217-230

Sen A, Smith T E, 1995 Gravity Models of Spatial Interaction Behavior (Springer, Berlin)

Smith T E, 1984, "Testable characterizations of gravity models" Geographical Analysis 16 74-94

Smith T E, Hsieh S-H, 1997a, "Gravity-type interactive Markov models-part I: a programming formulation of steady states" Journal of Regional Science $37653-682$

Smith T E, Hsieh S-H, 1997b, "Gravity-type interactive Markov models-part II: Lyapunov stability of steady states" Journal of Regional Science $37683-708$

Smith T R, Clayton C, 1978, "Transitivity, spatially separable utility functions, and United States migration streams, 1935-1970" Environment and Planning A 10399 - 414

Theil H, 1972 Statistical Decomposition Analysis (North-Holland, Amsterdam)

Van Campenhout J M, Cover T M, 1981, "Maximum entropy and conditional probability", IEEE Transactions on Information Theory $27483-489$

Weidlich W, Haag G (Eds), 1988 Interregional Migration (Springer, Berlin)

Willekens F J, 1983, "Specification and calibration of spatial interaction models: a contingencytable perspective and an application to intra-urban migration in Rotterdam" Tijdschrift voor Economische en Sociale Geografie $74239-252$

Wilson A G, 1974 Urban and Regional Models in Geography and Planning (John Wiley, New York) 


\section{APPENDIX}

\section{Proof of theorem 1}

(b) $\Rightarrow$ (a) is trivial. Defining $a_{j}:=\alpha_{j} / \beta_{j}$, and $c_{j k}:=\beta_{j} \beta_{k} \gamma_{j k}$ shows (a) $\Rightarrow$ (b). Conversely, suppose (b) holds and define $n_{j k}^{\mathrm{g}}:=\left(n_{j k} n_{k j}\right)^{1 / 2}$ and $u_{j}$ by equation (5). Direct substitution gives $n_{j k}^{\mathrm{g}} \exp \left(u_{k}-u_{j}\right)=n_{j k}$, thus showing (b) $\Rightarrow$ (f). Also (f) $\Rightarrow$ (a) by construction. (b) $\Rightarrow$ (g) and (g) $\Rightarrow$ (a) is shown analogously. The definition $w_{j k}:=n_{j k} / n_{j \bullet}$ entails the equivalence (d) $\Leftrightarrow(\mathrm{e})$. To show (f) $\Rightarrow(\mathrm{h})$, one can check that $\pi_{j}$, as defined in equation (6), is a normalized distribution satisfying

$$
\sum_{k=1}^{m} \pi_{j} w_{j k}=\pi_{k}
$$

with $w_{j k}:=n_{j k} / n_{j \bullet}$, and $\pi_{j} w_{j k}=\pi_{k} w_{k j}$. The equivalence (e) $\Leftrightarrow(\mathrm{h})$ is known as Kolmogorov's reversibility criterion in the study of reversible Markov chains (for a proof, see, for example, Kijima, 1997, page 60). Finally, the proof of the log-linear expression (c) for quasi-symmetry is standard (for example, see Bishop et al, 1975, page 286; Andersen, 1990, page 322). Statements $\left(\mathrm{e}^{\prime}\right)$ to $\left(\mathrm{h}^{\prime}\right)$ are proved in an analogous way.

\section{Proof of theorem 2}

1. By construction, $n_{j k}=n \rho_{j}^{*} w_{j k}$. Thus, $n_{j k} / n_{k j}=\rho_{j}^{*} \pi_{k} / \rho_{k}^{*} \pi_{j}$, using the quasi-symmetric property $w_{j k} / w_{k j}=\pi_{k} / \pi_{j}$. Proceeding similarly with $n_{j k}=n \rho_{k} w_{k j}^{*}$ yields alternatively $n_{j k} / n_{k j}=\rho_{k} \pi_{j}^{*} / \rho_{j} \pi_{k}^{*}$. Thus

$$
\frac{\rho_{j}^{*} \pi_{k}}{\rho_{k}^{*} \pi_{j}}=\frac{\rho_{k} \pi_{j}^{*}}{\rho_{j} \pi_{k}^{*}}, \quad \text { and therefore } \frac{\pi_{j} \pi_{j}^{*}}{\rho_{j} \rho_{j}^{*}}=\frac{\pi_{k} \pi_{k}^{*}}{\rho_{k} \rho_{k}^{*}}=\text { cst }=: c>0 .
$$

2. The proof follows from (1) as well as the unicity of the stationary distributions. For instance, suppose $\pi_{j}=\pi_{j}^{*}$. Then $\pi_{j}$ can be shown to be the stationary distribution of the transition matrix, $\alpha_{j k} / \alpha_{j_{\bullet}}$, where $\alpha_{j k}:=\sum_{j^{\prime}} \rho_{j^{\prime}}^{*} w_{j^{\prime} j} w_{j^{\prime} k}=\alpha_{k j}$, and $\alpha_{j_{\bullet}}=\rho_{j}$. On the other hand, $\alpha_{j k}$ being symmetric, $\alpha_{j_{\bullet}} / \alpha_{\bullet \bullet}=\rho_{j}$ is a stationary distribution of $\alpha_{j k} / \alpha_{j \bullet}$ as well. Unicity thus implies $\rho_{j}=\pi_{j}=\pi_{j}^{*}$, yielding in turn $\pi_{j}^{*}=\pi_{j}$.

3. Setting $n_{j k}=\alpha_{j} \beta_{k}$ and applying the definitions of section 2.1 shows $n_{j k}=n \rho_{j}^{*} \rho_{k}$ and, therefore, $\rho_{k}=\pi_{k}$. Then $w_{k j}^{*}=\rho_{j}^{*}$, which shows $\pi_{j}^{*}=\rho_{j}^{*}$.

\section{Proof of theorem 3}

1. Equation (9) makes it clear the push - pull term is the only source of asymmetry in the representation of flows. Thus, $n_{j k}=n_{k j}$, iff $u_{j}=$ cst $=0, \forall j$.

2. Similarly, the accessibilities, $g_{j k}$, are the only terms in equation (9) which do not decompose as a product of the form $\alpha_{j} \beta_{k}$. Thus $g_{j k}=$ constant $=1, \forall j, k$ whenever flows $n_{j k}$ are independent.

\section{ML estimation}

Writing the quasi-symmetric parameters as $\lambda_{j k}=a_{j} c_{j k}$ yields together with the Poisson distribution (14) the log-likelihood expression

$$
\ln \mathrm{P}(n \mid \lambda)=\sum_{j k}\left(-a_{j} c_{j k}+n_{j k} \ln a_{j} c_{j k}\right)-\sum_{j k} \ln \left(n_{j k} !\right) .
$$

Maximization of equation (A2) under the constraint $c_{j k}=c_{k j}$ is performed by incorporating the Lagrange multiplier, $\sum_{j k} \epsilon_{j k} c_{j k}=0$, where $\epsilon_{j k}=-\epsilon_{k j}$. The result is

$$
a_{j}=\frac{n_{j \bullet}}{c_{j \bullet}}, \quad c_{j k}=\frac{n_{j k}+n_{k j}}{a_{j}+a_{k}},
$$


or equivalently

$$
\lambda_{j \bullet}^{\mathrm{ML}}=n_{j \bullet}, \quad \lambda_{j k}^{\mathrm{ML}}+\lambda_{k j}^{\mathrm{ML}}=n_{j k}+n_{k j} .
$$

Thus the solution, $\lambda_{j k}^{\mathrm{ML}}=a_{j}^{(\infty)} c_{j k}^{(\infty)}$, can be obtained numerically by iteration:

$$
a_{j}^{(r)}:=\frac{n_{j \bullet}}{c_{j \bullet}^{(r)}}, \quad c_{j k}^{(r+1)}=\frac{n_{j k}+n_{k j}}{a_{j}^{(r)}+a_{k}^{(r)}},
$$

with some initial condition such as $a_{j}^{(0)}=1 / m$.

The log-likelihood $\chi^{2}$ is

$$
-2 \ln \frac{\ln \mathrm{P}\left(n \mid \lambda^{\mathrm{ML}}\right)}{\ln \mathrm{P}\left(n \mid \lambda^{\max }\right)}=2 n_{\bullet \bullet} \mathrm{K}\left(f \| p^{\mathrm{ML}}\right),
$$

where $\lambda^{\mathrm{ML}}$ (or $\lambda^{\mathrm{max}}$ ) represent the maximum-likelihood parameters with (without) the quasi-symmetry constraint. The last identity follows from the definition $p_{j k}:=\lambda_{j k} / \lambda_{\text {.. }}$, and the properties $\lambda_{j k}^{\max }=n_{j k}$, and $\lambda_{\bullet \bullet}^{\mathrm{ML}}=n_{\bullet \bullet}$; it demonstrates the equivalence between problem (21) [maximizing $\mathrm{P}(n \mid \lambda)$ over all quasi-symmetric $\lambda$ ] and problem (28) [minimizing $\mathrm{K}(f \| p)$ over all quasi-symmetric $p$ ].

\section{MDI estimation}

The MDI estimate is $\lambda_{j k}^{\mathrm{MDI}}=n_{\bullet \bullet} p_{j k}^{\mathrm{MDI}}$, where $p_{j k}^{\mathrm{MDI}}$ solves problem (27) under the constraint $p_{\bullet}^{\mathrm{MDI}}=1$. Writing $p_{j k}=\alpha_{j} \gamma_{j k}$ and proceeding as before yields

$$
\sum_{k} p_{j k} \ln \frac{p_{j k}}{f_{j k}}=K^{\mathrm{MDI}} p_{j \bullet}, \quad p_{j k} \ln \frac{p_{j k}}{f_{j k}}+p_{k j} \ln \frac{p_{k j}}{f_{k j}}=K^{\mathrm{MDI}}\left(p_{j k}+p_{k j}\right),
$$

where $f_{j k}:=n_{j k} / n_{\bullet .}$, and $K^{\mathrm{MDI}} \geqslant 0$ is the minimum value of equation (27), satisfying $K_{\mathrm{MDI}}=0$, iff $f_{j k}$ is quasi-symmetric, in which case $p_{j k}^{\mathrm{MDI}}=f_{j k}$ is, of course, the solution.

Equation (50) suggests that we should define $q_{j k}:=p_{j k} \exp \left(-K^{\mathrm{MDI}}\right)$. By quasisymmetry, $q_{j k}$ is of the form $q_{j k}=a_{j} c_{j k}$, with $c_{j k}=c_{k j}$. Equation (50) yields

$$
a_{j}=\prod_{k}\left(\frac{f_{j k}}{c_{j k}}\right)^{\frac{c_{j k}}{c_{j \bullet}}}, \quad c_{j k}=\left(\frac{f_{j k}}{a_{j}}\right)^{\frac{a_{j}}{a_{j}+a_{k}}}\left(\frac{f_{k j}}{a_{k}}\right)^{\frac{a_{k}}{a_{j}+a_{k}}} .
$$

The solution $\lambda_{j k}^{\mathrm{MDI}}=n_{. \bullet} \exp \left(K^{\mathrm{MDI}}\right) a_{j}^{(\infty)} c_{j k}^{(\infty)}$ can be obtained iteratively as

$$
a_{j}^{(r+1)}=\prod_{k}\left(\frac{f_{j k}}{c_{j k}^{(r)}}\right)^{\frac{c_{j k}^{(r)}}{c_{j \cdot}^{(r)}}}, \quad c_{j k}^{(r+1)}=\left(\frac{f_{j k}}{a_{j}^{(r)}}\right)^{\frac{a_{j}^{(r)}}{a_{j}^{(r)}+a_{k}^{(r)}}}\left(\frac{f_{k j}}{a_{k}^{(r)}}\right)^{\frac{a_{k}^{(r)}}{a_{j}^{(r)}+a_{k}^{(r)}}},
$$

with $K^{\mathrm{MDI}}:=-\ln \sum_{j k} a_{j}^{(\infty)} c_{j k}^{(\infty)}\left[\right.$ because $\left.\sum_{j k} q_{j k}^{(\infty)}=\exp \left(-K^{\mathrm{MDI}}\right)\right]$, together with an initial condition such as $a_{j}^{(0)}=1 / m$.

\section{Maximum-entropy estimation}

Minimizing equation (29) under the marginal constraints $p_{i \bullet}^{\mathrm{ME}}=f_{i \bullet}$ [constraint (2)] and $p_{\bullet j}^{\mathrm{ME}}=f_{\bullet j}$ [constraint (3)] as well as the trace constraint $\operatorname{tr}\left(p^{\mathrm{ME}}\right):=\sum_{j} p_{j j}^{\mathrm{ME}}=\operatorname{tr}(f)$ [constraint (4)] with uniform prior, $p_{j k}^{0}$, yields a solution of the form (31), reparametrizable as $p_{j k}^{\mathrm{ME}}=\left(A+B \delta_{j k}\right) \alpha_{j} \beta_{k}$, with $\alpha_{\bullet}=\beta_{\bullet}=1$. As predicted by the maximum-entropy lemma for quasi-symmetry, $p_{j k}^{\mathrm{ME}}$ is automatically quasi-symmetric. The conditions to be satisfied by the new parameters are $\alpha_{j}\left(A+B \beta_{j}\right)=f_{j \bullet}$, 
$\beta_{k}\left(A+B \alpha_{k}\right)=f_{\bullet k}$, and $(A+B)(\alpha, \beta)=\operatorname{tr}(f)$. Solutions can be obtained iteratively as

$$
\begin{aligned}
A^{(r)} & =\frac{1-\operatorname{tr}(f)}{1-\left(\alpha^{(r)}, \beta^{(r)}\right)}, & B^{(r)} & =\frac{1-A^{(r)}}{\left(\alpha^{(r)}, \beta^{(r)}\right)}, \\
\alpha_{j}^{(r+1)} & =\frac{f_{\text {• }}}{A^{(r)}+B^{(r)} \beta_{j}^{(r)}}, & \beta_{k}^{(r+1)} & =\frac{f_{\bullet k}}{A^{(r)}+B^{(r)} \alpha_{k}^{(r)}},
\end{aligned}
$$

with initial conditions $\alpha_{j}^{(0)}=f_{j \bullet}$, and $\beta_{k}^{(0)}=f_{\bullet k}$.

\section{Generalized $s$-divergence estimation}

The generalized $s$-divergence estimate is $\lambda_{j k}^{s}=n_{\bullet .} p_{j k}^{s}$, where $p_{j k}^{s}$ solves problem (33) under the constraint $p_{\bullet}^{s}=1$. Writing $p_{j k}=a_{j} c_{j k}$ and proceeding as before yields equation (34), or equivalently

$$
a_{j}^{s+1}=\frac{1}{B_{s} c_{j \bullet}} \sum_{k} \frac{f_{j k}^{s+1}}{c_{j k}^{s}}, \quad c_{j k}^{s+1}=\frac{1}{B_{s}\left(a_{j}+a_{k}\right)}\left(\frac{f_{j k}^{s+1}}{a_{j}^{s}}+\frac{f_{k j}^{s+1}}{a_{k}^{s}}\right) .
$$

The solution $\lambda_{j k}^{s}=n_{\bullet \bullet}^{(\infty)} c_{j k}^{(\infty)}$ can be obtained iteratively as

$$
\begin{aligned}
& a_{j}^{(r+1)}=\left(\frac{1}{B^{(r)} c_{j \bullet}^{(r)}} \sum_{k} \frac{f_{j k}^{s+1}}{c_{j k}^{(r)^{s}}}\right)^{1 /(s+1)}, \\
& c_{j k}^{(r+1)}=\left[\frac{1}{B^{(r)}\left(a_{j}^{(r)}+a_{k}^{(r)}\right)}\left(\frac{f_{j k}^{s+1}}{a_{j}^{(r)^{s}}}+\frac{f_{k j}^{s+1}}{a_{k}^{(r)^{s}}}\right)\right]^{1 /(s+1)},
\end{aligned}
$$

where $B^{(r)}:=s(s+1) \mathrm{I}_{s}\left(f: p^{(r)}\right)+1$, and $p_{j k}^{(r)}:=a_{j}^{(r)} c_{j k}^{(r)}$.

Note the estimation of the diagonal parameters to be generally not trivial, because equation (A12) yields $p_{j j}^{s}=f_{j j} B_{s}^{-1 /(s+1)}$ : as $B_{s}>1$, for $s>0$, or $s<-1$, one gets $p_{j j}^{s}<f_{i j}$, for $s>0$, and $p_{j j}^{s}>f_{j j}$, for $s<-1$. Similarly, $p_{j j}^{s}>f_{j j}$, for $-1<s<0$. In the case $s=0$, one recovers the $\mathrm{ML}$ result $p_{j j}^{\mathrm{ML}}=f_{j j}$. For $s=-1$, one gets $p_{j j}^{\mathrm{MDI}}=f_{j j} \exp K^{\mathrm{MDI}}$, which could have been obtained directly from equation (A7).

\section{Least squares estimation}

Proceeding as before, minimizing equation (35) under the constraint $\lambda_{j k}=a_{j} c_{j k}$, with $c_{j k}=c_{k j}$, yields

$$
\left(a_{j}^{\mathrm{LS}^{2}}+a_{k}^{\mathrm{LS}^{2}}\right) c_{j k}=a_{j}^{\mathrm{LS}} n_{j k}+a_{k}^{\mathrm{LS}} n_{k j}, \quad a_{j}^{\mathrm{LS}} \sum_{l=1}^{m} c_{j l}^{\mathrm{LS}^{2}}=\sum_{l=1}^{m} c_{j l}^{\mathrm{LS}} n_{j l},
$$

which demonstrates equation (36). The solution $\lambda_{j k}^{\mathrm{LS}}=a_{j}^{(\infty)} c_{j k}^{(\infty)}$ can be obtained iteratively as

$$
a_{j}^{\mathrm{LS}(r)}=\frac{\sum_{l=1}^{m} c_{j l}^{\mathrm{LS}(\mathrm{r})} n_{j l}}{\sum_{l=1}^{m} c_{j l}^{\mathrm{LS}(r)^{2}}}, \quad c_{j l}^{\mathrm{LS}(r+1)}=\frac{a_{j}^{\mathrm{LS}(r)} n_{j k}+a_{k}^{\mathrm{LS}(r)} n_{k j}}{a_{j}^{\mathrm{LS}(r)^{2}}+a_{k}^{\mathrm{LS}(r)^{2}}} .
$$

\section{Logarithmic least squares estimation}

Proceeding as before, minimizing equation (37) under the constraint $\lambda_{j k}=a_{j} c_{j k}$, with $c_{j k}=c_{k j}$, yields

$$
\ln \lambda_{j k}^{\mathrm{LLS}}+\ln \lambda_{k j}^{\mathrm{LLS}}=\ln n_{j k}+\ln n_{k j}, \quad \sum_{k=1}^{m} \ln \lambda_{j k}^{\mathrm{LLS}}=\sum_{k=1}^{m} \ln n_{j k},
$$

which demonstrates equation (38). 
